\title{
An Improved Family of Estimators of Finite Population Mean Using Information on an Auxiliary Variable in Sample Surveys
}

\author{
H. P. SINGH AND A. YADAV
}

\begin{abstract}
In this paper we have suggested a family of estimators of the population mean using auxiliary information in sample surveys. The bias and mean squared error of the proposed class of estimators have been obtained under large sample approximation. We have derived the conditions for the parameters under which the proposed class of estimators has smaller mean squared error than the sample mean, ratio, product, regression estimator and the two parameter ratio-product-ratio estimators envisaged by Chami et al (2012). An empirical study is carried out to demonstrate the performance of the proposed class of estimators over other existing estimators.
\end{abstract}

\section{Mathematics Subject Classification 2000: $62 \mathrm{D05}$}

Keywords: Study variable, Auxiliary variable, Bias, Mean squared Error

\section{INTRODUCTION}

Survey statisticians are always seeking to estimate the population parameters such as mean or total with greater precision. The precision of an estimate can be enhanced by using auxiliary information at the estimation stage. The ratio and regression methods of estimation are two classical methods of estimation in survey sampling which use information about auxiliary variable. A large number of modifications of ratio method of estimation is available in the literature, for instance see Singh, H.P. (1986), Singh, S. (2003) and the references cited therein. We have also made an effort to develop an efficient class of estimators for population mean using auxiliary information at the estimation stage along with its properties.

Consider a finite population $\left(U=U_{1}, U_{2}, \ldots, U_{N}\right)$ of $N$ distinct and identifiable units. Let $(y, x)$ be the (study, auxiliary) variables taking values $\left(y_{i}, x_{i}\right)$ measured on $U_{i}, i=1,2, \ldots, N$. Let $(\bar{Y}, \bar{X})$ be the population means of the variables $(y, x)$ respectively. It is assumed that the population mean $\bar{X}$ of the auxiliary variable $x$ is 
known. It is desired to estimate the population mean $\bar{Y}$ of the study variable $y$ using the information on the population mean $\bar{X}$ of the auxiliary variable $x$ and on the basis of a random sample selected from the population $U$ For estimating the population mean $\bar{Y}$ of the study variable $y$ a simple random sample of size $n$ is drawn without replacement from $U$ Let $(\bar{y}, \bar{x})$ be the sample means of the variables $(y, x)$ respectively based on $n$ observations.

The usual unbiased estimator of the population means $\bar{Y}$ (which does not utilize auxiliary information) is defined by

$$
t_{0}=\bar{y}=\frac{1}{n} \sum_{i=1}^{n} y_{i}
$$

with the variance / mean squared error (MSE):

$$
\begin{aligned}
\operatorname{MSE}(\bar{y})= & \frac{(1-f)}{n} \bar{Y}^{2} C_{y}^{2} \\
& =\frac{(1-f)}{n} S_{y}^{2}
\end{aligned}
$$

where $f=\frac{n}{N}, C_{y}=\frac{S_{y}}{\bar{Y}}$ and $S_{y}^{2}=\frac{1}{N-1} \sum_{i=1}^{N}\left(y_{i}-\bar{Y}\right)^{2}$.

The classical ratio and product estimators for the population mean $\bar{Y}$ are respectively defined by

$$
\bar{y}_{R}=\bar{y}\left(\frac{\bar{X}}{\bar{x}}\right) \text { (ratio estimator) }
$$

and

$$
\bar{y}_{p}=\bar{y}\left(\frac{\bar{x}}{\bar{X}}\right) \text { (product estimator) }
$$

To the first degree of approximation the mean squared errors of the ratio estimator $\bar{y}_{R}$ and the product estimator $\bar{y}_{p}$ are respectively given by

$$
\begin{aligned}
& \operatorname{MSE}\left(\bar{y}_{R}\right)=\frac{(1-f)}{n} \bar{Y}^{2}\left[C_{y}^{2}+C_{x}^{2}(1-2 C)\right], \\
& \operatorname{MSE}\left(\bar{y}_{p}\right)=\frac{(1-f)}{n} \bar{Y}^{2}\left[C_{y}^{2}+C_{x}^{2}(1+2 C)\right],
\end{aligned}
$$


where $C_{x}=\frac{S_{x}}{\bar{X}}, C=\frac{\rho C_{y}}{C_{x}}, S_{x}^{2}=\frac{1}{N-1} \sum_{i=1}^{N}\left(x_{i}-\bar{X}\right)^{2}, \rho=\frac{S_{x y}}{S_{x} S_{y}}$ is the correlation coefficient between $y$ and $x ; S_{x y}=\frac{1}{N-1} \sum_{i=1}^{N}\left(x_{i}-\bar{X}\right)\left(y_{i}-\bar{Y}\right)$.

Murthy (1964) and Sahai and Ray (1980) compared the relative precision of the estimators $\bar{y}, \bar{y}_{R}$ and $\bar{y}_{p}$; showed that the ratio estimator $\bar{y}_{R}$, sample mean $\bar{y}$, and product estimator $\bar{y}_{p}$ are most efficient when $C>\frac{1}{2},-\frac{1}{2} \leq C \leq \frac{1}{2}$ and $C<-\frac{1}{2}$ respectively. In other words, when there is positive (high) correlation between the study variable $y$ and the auxiliary variable $x$, the ratio estimator $\bar{y}_{R}$ can be employed. On the other hand, if the correlation between $y$ and $x$ is negative (high) the product method of estimation is employed quite effectively. When the variables $(y, x)$ show a weak correlation only, then the sample mean $\bar{y}$ is to be used in practice. Several authors have given the modified versions of ratio and product estimators along with their properties under large sample approximation, for instance, see Srivastava (1967), Reddy (1973, 1974, 1978), Sahai and Ray (1980), Gupta (1971), Singh and Ruiz Espejo (2003), Chami et al (2012), Singh H.P. (1986), Singh, S. (2003) and references cited there in.

In this paper we have made an effort to suggest a class of estimators for population mean $\bar{Y}$ using known population mean $\bar{X}$ of the auxiliary variable $x$. The properties of the proposed class of estimators re studied to the first degree of approximation. Theoretical and empirical comparisons are carried out to show the superiority of the suggested class of estimators over existing estimators. 


\section{THE SUGGESTED FAMILY OF ESTIMATORS}

For estimating the population mean $\bar{Y}$ of the study variable $y$, we proposed the following family of estimators:

$$
T=\bar{y}\left[\alpha_{1}\left\{\frac{(1-\beta) \bar{x}+\beta \bar{X}}{\beta \bar{x}+(1-\beta) \bar{X}}\right\}^{\delta}+\alpha_{2}\left\{\frac{\beta \bar{x}+(1-\beta) \bar{X}}{(1-\beta) \bar{x}+\beta \bar{X}}\right\}\right]
$$

where $\left(\alpha_{1}, \alpha_{2}\right)$ are suitably chosen weights whose sum need not be unity; and $(\beta, \delta)$ are real constants and $\beta$ may also take the values of the known parameters such as $C_{\mathrm{x}}$ (Coefficient of variation), $\beta_{1}(x)$ (coefficient of skewness), $\beta_{2}(x)$ (coefficient of kurtosis) and $S_{\mathrm{x}}$ (Standard deviation) associated with the auxiliary variable $x$ and the value of $\rho$ the correlation coefficient between $y$ and $x$. We note that for different values of $\left(\alpha_{1}, \alpha_{2}, \beta, \delta\right)$ a large number of estimators can be identified as a member of the suggested family of estimators.

Some members of the suggested family of estimators $T$ are presented in Table 2.1.

Table 2.1 - Some members of the proposed family of estimators $T$.

\begin{tabular}{|c|c|c|c|c|c|}
\hline \multirow{2}{*}{ S.no. } & Estimator & \multicolumn{3}{|c|}{ Values of constants } \\
\cline { 3 - 5 } 1. & $\alpha_{1}=\alpha_{1} \bar{y}+\alpha_{2} \bar{y}\left(\frac{\bar{x}}{\bar{X}}\right)$ & $\alpha_{2}$ & $\alpha_{2}$ & 0 & 1 \\
\hline 2. & Upadhayaya et al (1985) estimator & & & \\
\hline 3. & $T_{(2)}=\alpha_{1} \bar{y}+\alpha_{2} \bar{y}\left(\frac{\bar{X}}{\bar{x}}\right)$ & $\alpha_{1}$ & $\alpha_{2}$ & 0 & 0 \\
\hline 4. & $T_{(3)}=\alpha_{1} \bar{y}\left(\frac{\bar{X}}{\bar{x}}\right)^{1 / 2}+\alpha_{2} \bar{y}\left(\frac{\bar{x}}{\bar{X}}\right)$ & $\alpha_{1}$ & $\alpha_{2}$ & $1 / 2$ & 1 \\
\hline 5. & $T_{(4)}=\alpha_{1} \bar{y}\left(\frac{\bar{X}}{\bar{x}}\right)^{2}+\alpha_{2} \bar{y}\left(\frac{\bar{x}}{\bar{X}}\right)$ & $\alpha_{1}$ & $\alpha_{2}$ & 1 & 1 \\
\hline & $T_{(5)}=\alpha_{1} \bar{y}\left(\frac{\bar{X}}{\bar{x}}\right)^{2}+\alpha_{2} \bar{y}\left(\frac{\bar{x}}{\bar{X}}\right)$ & $\alpha_{1}$ & $\alpha_{2}$ & 2 & 1 \\
\hline
\end{tabular}




\begin{tabular}{|c|c|c|c|c|c|}
\hline \multirow{2}{*}{ S.no. } & \multirow{2}{*}{ Estimator } & \multicolumn{4}{|c|}{ Values of constants } \\
\hline & & $\alpha_{1}$ & $\alpha_{2}$ & $\delta$ & $\beta$ \\
\hline 6. & $\begin{aligned} T_{(6)}=\alpha_{1} \bar{y} & \left\{\frac{\left(1-C_{x}\right) \bar{x}+C_{x} \bar{X}}{C_{x} \bar{x}+\left(1-C_{x}\right) \bar{X}}\right\}^{1 / 2} \\
& +\alpha_{2} \bar{y}\left\{\frac{C_{x} \bar{x}+\left(1-C_{x}\right) \bar{X}}{\left(1-C_{x}\right) \bar{x}+C_{x} \bar{X}}\right.\end{aligned}$ & $\alpha_{1}$ & $\alpha_{2}$ & $1 / 2$ & 1 \\
\hline 7. & $\begin{aligned} T_{(7)}=\alpha_{1} \bar{y} & \left\{\frac{\left(1-C_{x}\right) \bar{x}+C_{x} \bar{X}}{C_{x} \bar{x}+\left(1-C_{x}\right) \bar{X}}\right\} \\
& +\alpha_{2} \bar{y}\left\{\frac{C_{x} \bar{x}+\left(1-C_{x}\right) \bar{X}}{\left(1-C_{x}\right) \bar{x}+C_{x} \bar{X}}\right\}\end{aligned}$ & $\alpha_{1}$ & $\alpha_{2}$ & 1 & 1 \\
\hline 8. & $\begin{aligned} T_{(8)}=\alpha_{1} \bar{y} & \left\{\frac{\left(1-C_{x}\right) \bar{x}+C_{x} \bar{X}}{C_{x} \bar{x}+\left(1-C_{x}\right) \bar{X}}\right\}^{2} \\
& +\alpha_{2} \bar{y}\left\{\frac{C_{x} \bar{x}+\left(1-C_{x}\right) \bar{X}}{\left(1-C_{x}\right) \bar{x}+C_{x} \bar{X}}\right.\end{aligned}$ & $\alpha_{1}$ & $\alpha_{2}$ & 2 & 1 \\
\hline 9. & $T_{(9)}=\alpha_{1} \bar{y}+\alpha_{2} \bar{y}\left\{\frac{C_{x} \bar{x}+\left(1-C_{x}\right) \bar{X}}{\left(1-C_{x}\right) \bar{x}+C_{x} \bar{X}}\right.$ & $\alpha_{1}$ & $\alpha_{2}$ & 0 & $C_{\mathrm{x}}$ \\
\hline 10. & $\begin{aligned} T_{(10)}=\alpha_{1} \bar{y} & \left\{\frac{(1-\rho) \bar{x}+\rho \bar{X}}{\rho \bar{x}+(1-\rho) \bar{X}}\right\} \\
& +\alpha_{2} \bar{y}\left\{\frac{\rho \bar{x}+(1-\rho) \bar{X}}{(1-\rho) \bar{x}+\rho \bar{X}}\right\}\end{aligned}$ & $\alpha_{1}$ & $\alpha_{2}$ & 1 & $\rho$ \\
\hline 11. & $\begin{aligned} & T_{(11)}=\alpha_{1} \bar{y}\left\{\frac{(1-\rho) \bar{x}+\rho \bar{X}}{\rho \bar{x}+(1-\rho) \bar{X}}\right\} \\
&+\alpha_{2} \bar{y}\left\{\frac{\rho \bar{x}+(1-\rho) \bar{X}}{(1-\rho) \bar{x}+\rho \bar{X}}\right\}\end{aligned}$ & $\alpha_{1}$ & $\alpha_{2}$ & 2 & $\rho$ \\
\hline 12. & $\begin{aligned} T_{(12)}=\alpha_{1} \bar{y} & \left\{\frac{\left(1-C_{y}\right) \bar{x}+C_{y} \bar{X}}{C_{y} \bar{x}+\left(1-C_{y}\right) \bar{X}}\right\} \\
& +\alpha_{2} \bar{y}\left\{\frac{C_{y} \bar{x}+\left(1-C_{y}\right) \bar{X}}{\left(1-C_{y}\right) \bar{x}+C_{y} \bar{X}}\right.\end{aligned}$ & $\alpha_{1}$ & $\alpha_{2}$ & 2 & $C_{\mathrm{y}}$ \\
\hline
\end{tabular}


We can also choose $\beta=\frac{n}{N}, \frac{n}{N-n}, \frac{f}{1-f}, \frac{(1-f)}{(1+f)}$.

for defining the estimators for population mean $\bar{Y}$ from the suggested family of estimators $T$.

\subsection{First-Degree Approximation to the Bias and Mean Squared Error}

To obtain the bias and mean squared error of the proposed estimator $T$, we write

$$
\bar{y}=\bar{Y}\left(1+e_{0}\right), \bar{x}=\bar{X}\left(1+e_{1}\right)
$$

Such that

$$
E\left(e_{0}\right)=E\left(e_{1}\right)=0
$$

and

$$
\begin{aligned}
& E\left(e_{0}^{2}\right)=\frac{(1-f)}{n} C_{y}^{2}, \\
& E\left(e_{1}^{2}\right)=\frac{(1-f)}{n} C_{x}^{2}, \\
& E\left(e_{0} e_{1}\right)=\frac{(1-f)}{n} C C_{x}^{2},
\end{aligned}
$$

where $C=\rho\left(\frac{C_{y}}{C_{x}}\right)$.

Now expressing (2.1) in terms of $e^{\prime} s$ we have

$$
\left.T=\bar{y}\left(1+e_{0}\right) \mid \alpha_{1}\left\{1+(1-\beta) e_{1}\right\}^{\delta}\left(1+\beta e_{1}\right)^{-\delta}+\alpha_{2}\left(1+\beta e_{1}\right)\left\{1+(1-\beta) e_{1}\right\}^{-1}\right\rfloor
$$

We assume that $\left|e_{1}\right|<\min .\left\{\frac{1}{|\beta|}, \frac{1}{|(1-\beta)|}\right\}$ so that we can expand $\left\{1+(1-\beta) e_{1}\right\}^{\delta}$, $\left(1+\beta e_{1}\right)^{-\delta},\left\{1+(1-\beta) e_{1}\right\}^{-1}$ as a series in powers of $e_{1}$. 
Chami et al (2012) have mentioned that $\min \cdot\left\{\frac{1}{|\beta|}, \frac{1}{|(1-\beta)|}\right\}$ attains its maximal value 2 at $\beta=\frac{1}{2}$. We get up to $O\left(e_{1}^{2}\right)$,

$$
T=\bar{Y}\left(1+e_{0}\right)\left[\begin{array}{l}
\alpha_{1}\left\{1+\delta(1-2 \beta) e_{1}+\frac{\delta(1-2 \beta)}{2}[\delta(1-2 \beta)-1]\right\}+ \\
\alpha_{2}\left\{1-(1-2 \beta) e_{1}+(1-\beta)(1-2 \beta) e_{1}^{2}\right\}+O\left(e_{1}^{3}\right)
\end{array}\right]
$$

We assume that the sample size $n$ is large enough to make $\left|e_{1}\right|$ so small that contributions from powers of $e_{1}$ degree higher than two are negligible. By powers retaining up to $e_{1}^{2}$, we get

$$
T=\bar{Y}\left[\begin{array}{l}
\alpha_{1}\left\{1+e_{0}+\delta(1-2 \beta) e_{1}+\delta(1-2 \beta) e_{0} e_{1}+\frac{\delta(1-2 \beta)(\delta(1-2 \beta)-1)}{2} e_{1}^{2}\right\} \\
\alpha_{2}\left\{1+e_{0}-(1-2 \beta) e_{1}-(1-2 \beta) e_{0} e_{1}+(1-\beta)(1-2 \beta) e_{1}^{2}\right\}
\end{array}\right]
$$

Or

$$
(T-\bar{Y})=\bar{Y}\left[\begin{array}{l}
\alpha_{1}\left\{1+e_{0}+\delta(1-2 \beta) e_{1}+\delta(1-2 \beta) e_{0} e_{1}+\frac{\delta(1-2 \beta)(\delta(1-2 \beta)-1)}{2} e_{1}^{2}\right\} \\
\alpha_{2}\left\{1+e_{0}-(1-2 \beta) e_{1}-(1-2 \beta) e_{0} e_{1}+(1-\beta)(1-2 \beta) e_{1}^{2}\right\}-1
\end{array}\right]
$$

Taking expectation of both sides of (2.4) we get the bias of $T$ to the first degree of approximation as

$$
B(T)=\bar{Y}\left[\begin{array}{l}
\alpha_{1}\left\{1+\frac{\delta(1-2 \beta)}{2} \frac{(1-f)}{n} C_{x}^{2}(\delta(1-2 \beta)+2 C-1)\right\} \\
+\alpha_{2}\left\{1+(1-2 \beta) \frac{(1-f)}{n} C_{x}^{2}(1-\beta-C)\right\}-1
\end{array}\right]
$$

The bias of $T$ would be zero if the sample size $n$ is sufficiently large and $\alpha_{1}+\alpha_{2}=1$. The bias of the estimators belonging to the proposed class of estimators can be easily obtained from (2.5) just by putting the suitable values of the constants $\left(\alpha_{1}, \alpha_{2}, \alpha, \beta, \delta\right)$.

Squaring both sides of (2.4) and neglecting terms of $e^{\text {' }} s$ having power greater than two we have 


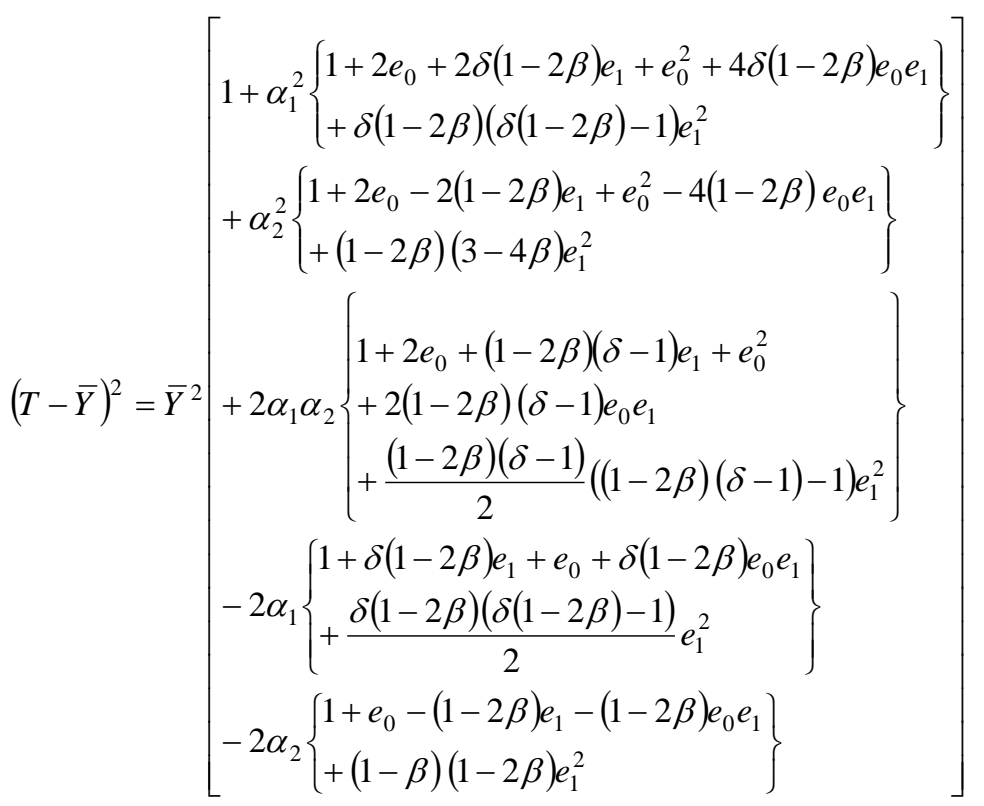

Taking expectation of both sides of (2.5) we get the mean squared error of the suggested class of estimators $T$ to the first degree of approximation as

$$
\operatorname{MSE}(T)=\bar{Y}^{2}\left\lfloor 1+\alpha_{1}^{2} A_{1}+\alpha_{2}^{2} A_{2}+2 \alpha_{1} \alpha_{2} A_{3}-2 \alpha_{1} A_{4}-2 \alpha_{2} A_{5}\right\rfloor
$$

where

$$
\begin{aligned}
& A_{1}=\left[1+\frac{(1-f)}{n}\left\{C_{y}^{2}+C_{x}^{2} \delta(1-2 \beta)[2 \delta(1-2 \beta)+4 C-1]\right\},\right. \\
& A_{2}=\left[1+\frac{(1-f)}{n}\left\{C_{y}^{2}+C_{x}^{2}[(1-2 \beta)(3-4 \beta)-4(1-2 \beta) C]\right\},\right. \\
& A_{3}=\left[1+\frac{(1-f)}{n}\left\{C_{y}^{2}+C_{x}^{2} \frac{(1-2 \beta)(\delta-1)}{2}[(1-2 \beta)(\delta-1)+4 C-1]\right\},\right. \\
& A_{4}=\left[1+\frac{(1-f)}{2 n} \delta(1-2 \beta) C_{x}^{2}\{\delta(1-2 \beta)+2 C-1\}\right], \\
& A_{5}=\left[1+\frac{(1-f)}{n}(1-2 \beta) C_{x}^{2}(1-\beta-C)\right] .
\end{aligned}
$$

Differentiating (2.7) with respect to $\alpha_{1}$ and $\alpha_{2}$ and equating them to zero we have

$$
\left[\begin{array}{ll}
A_{1} & A_{2} \\
A_{3} & A_{2}
\end{array}\right]\left[\begin{array}{l}
\alpha_{1} \\
\alpha_{2}
\end{array}\right]=\left[\begin{array}{l}
A_{4} \\
A_{5}
\end{array}\right] .
$$


Solving (2.8) we get the optimum values of $\alpha_{1}$ and $\alpha_{2}$ as

$$
\left.\begin{array}{l}
\alpha_{1}=\frac{\left(A_{2} A_{4}-A_{3} A_{5}\right)}{\left(A_{1} A_{2}-A_{3}^{2}\right)}=\alpha_{10}(\text { say }) \\
\alpha_{2}=\frac{\left(A_{1} A_{5}-A_{3} A_{4}\right)}{\left(A_{1} A_{5}-A_{3}^{2}\right)}=\alpha_{20}(\text { say })
\end{array}\right\}
$$

Thus the resulting minimum $M S E$ of $T$ is given by

$$
M S E_{\min }(T)=\bar{Y}^{2}\left[1-\frac{\left(A_{2} A_{4}^{2}-2 A_{3} A_{4} A_{5}+A_{1} A_{5}^{2}\right)}{\left(A_{1} A_{2}-A_{3}^{2}\right)}\right]
$$

Now we state the following theorem.

THEOREM 2.1 - To the first degree of approximation,

$$
M S E_{\min }(T) \geq \bar{Y}^{2}\left[1-\frac{\left(A_{2} A_{4}^{2}-2 A_{3} A_{4} A_{5}+A_{1} A_{5}^{2}\right)}{\left(A_{1} A_{2}-A_{3}^{2}\right)}\right]
$$

with equality holding if

$$
\alpha_{1}=\alpha_{10} \text { and } \alpha_{2}=\alpha_{20} .
$$

Putting the optimum values $\alpha_{10}$ and $\alpha_{20}$ from (2.9) in place of $\alpha_{1}$ and $\alpha_{2}$ in (2.5) we get the resulting bias of $T$ is given by

$$
B_{0}(T)=-\bar{Y}\left[1-\frac{\left(A_{2} A_{4}^{2}-2 A_{3} A_{4} A_{5}+A_{1} A_{5}^{2}\right)}{\left(A_{1} A_{2}-A_{3}^{2}\right)}\right] .
$$

From (2.10), the relative minimum $M S E$ of $T$ is given by

$$
\begin{aligned}
R M S E_{\min }(T) & =\frac{M S E_{\text {min }}(T)}{\bar{Y}^{2}} \\
& =\left[1-\frac{\left(A_{2} A_{4}^{2}-2 A_{3} A_{4} A_{5}+A_{1} A_{5}^{2}\right)}{\left(A_{1} A_{2}-A_{3}^{2}\right)}\right] .
\end{aligned}
$$

From (2.11), the absolute relative resulting bias of $T$ is given by

$$
A R B_{0}(T)=\left|\frac{B_{0}(T)}{\bar{Y}}\right|=\left[1-\frac{\left(A_{2} A_{4}^{2}-2 A_{3} A_{4} A_{5}+A_{1} A_{5}^{2}\right)}{\left(A_{1} A_{2}-A_{3}^{2}\right)}\right] \text {. }
$$


Thus it follows from (2.12) and (2.13) that

$$
R M S E_{\min }(T)=A R B_{0}(T) .
$$

which follows that at the optimum condition the $M S E$ of $T$ is least as well as absolute relative resulting bias is reduced.

\subsection{The Sub Class of Estimators of $T$}

If we set $\alpha_{2}=0$ in (2.1) we get a subclass of estimators of the class of estimators $T$ of population mean $\bar{Y}$ as

$$
T_{1}=\alpha_{1} \bar{y}\left\{\frac{(1-\beta) \bar{x}+\beta \bar{X}}{\beta \bar{x}+(1-\beta) \bar{X}}\right\}^{\delta}
$$

\begin{tabular}{|c|c|c|c|c|}
\hline \multirow{2}{*}{ S.no. } & \multirow{2}{*}{ Estimator } & \multicolumn{3}{|c|}{ Values of constants } \\
\hline & & $\alpha$ & $\delta$ & $\beta$ \\
\hline 1. & $T_{1(1)}=\alpha_{1} \bar{y}\left(\frac{\bar{X}}{\bar{x}}\right)^{1 / 2}$ & $\alpha_{1}$ & $1 / 2$ & 1 \\
\hline 2. & $\begin{array}{c}T_{1(2)}=\alpha_{1} \bar{y} \frac{\bar{X}}{\bar{x}}=\bar{y}_{S P} \\
\text { Srivastava (1974) and Prasad } \\
\text { (1989) estimator }\end{array}$ & $\alpha_{1}$ & 1 & 1 \\
\hline 3. & $T_{1(3)}=\alpha_{1} \bar{y}\left\{\frac{\left(1-C_{x}\right) \bar{x}+C_{x} \bar{X}}{C_{x} \bar{x}+\left(1-C_{x}\right) \bar{X}}\right\}^{1 / 2}$ & $\alpha_{1}$ & $1 / 2$ & $C_{\mathrm{x}}$ \\
\hline 4. & $T_{1(4)}=\alpha_{1} \bar{y}\left\{\frac{(1-\rho) \bar{x}+\rho \bar{X}}{\rho \bar{x}+(1-\rho) \bar{X}}\right\}^{1 / 2}$ & $\alpha_{1}$ & $1 / 2$ & $C_{\mathrm{x}}$ \\
\hline 5. & $T_{1(5)}=\alpha_{1} \bar{y}\left\{\frac{(1-\rho) \bar{x}+\rho \bar{X}}{\rho \bar{x}+(1-\rho) \bar{X}}\right\}$ & $\alpha_{1}$ & 1 & $\rho$ \\
\hline
\end{tabular}

Table 2.2- Some members of the sub-class of estimators $T_{l}$ are shown in Table 2.2. 


\begin{tabular}{|c|c|c|c|c|}
\hline \multirow{2}{*}{ S.no. } & Estimator & \multicolumn{3}{|c|}{ Values of constants } \\
\cline { 3 - 5 } 6. & $T_{1(6)}=\alpha_{1} \bar{y}\left\{\frac{\left(1-C_{y}\right) \bar{x}+C_{y} \bar{X}}{C_{y} \bar{x}+\left(1-C_{y}\right) \bar{X}}\right\}$ & $\alpha_{1}$ & $1 / 2$ & $C_{\mathrm{y}}$ \\
\hline 7. & $T_{1(7)}=\alpha_{1} \bar{y}\left\{\frac{\left(1-C_{y}\right) \bar{x}+C_{y} \bar{X}}{C_{y} \bar{x}+\left(1-C_{y}\right) \bar{X}}\right\}$ & $\alpha_{1}$ & 1 & $C_{\mathrm{y}}$ \\
\hline 8. & $T_{1(8)}=\alpha_{1} \bar{y}\left\{\frac{(1-C) \bar{x}+C \bar{X}}{C \bar{x}+(1-C) \bar{X}}\right\}^{1 / 2}$ & $\alpha_{1}$ & $1 / 2$ & $C$ \\
\hline 9. & $T_{1(9)}=\alpha_{1} \bar{y}\left\{\frac{(1-C) \bar{x}+C \bar{X}}{C \bar{x}+(1-C) \bar{X}}\right\}^{2}$ & $\alpha_{1}$ & 1 & $C$ \\
\hline 10. & $T_{1(9)}=\alpha_{1} \bar{y}\left\{\frac{(1-C) \bar{x}+C \bar{X}}{C \bar{x}+(1-C) \bar{X}}\right\}^{2}$ & $\alpha_{1}$ & 2 & $C$ \\
\hline
\end{tabular}

Putting $\alpha_{2}=0$, in(2.5) and (2.7) we get the bias and MSE of $T_{1}$ to the first degree of approximation respectively as

$$
\begin{aligned}
& B\left(T_{1}\right)=\bar{Y}\left[\alpha_{1} A_{4}-1\right], \\
& \operatorname{MSE}\left(T_{1}\right)=\bar{Y}^{2}\left\lfloor 1+\alpha_{1}^{2} A_{1}-2 \alpha_{1} A_{4}\right\rfloor,
\end{aligned}
$$

where

$$
A_{4}=\left[1+\frac{(1-f)}{2 n} \delta(1-2 \beta) C_{x}^{2}\{\delta(1-2 \beta)+2 C-1\}\right] .
$$

The MSE of the sub class of estimators $T_{(1)}$ is minimum, when

$$
\begin{aligned}
\alpha_{1} & =A_{4} / A_{1} \\
& =\alpha_{10}^{*} .
\end{aligned}
$$

Thus the resulting bias and minimum $M S E$ of $T_{1}$ are respectively given by

$$
B_{0}\left(T_{1}\right)=-\bar{Y}\left(1-\frac{A_{4}^{2}}{A_{1}}\right)
$$

and 


$$
M S E_{\min }\left(T_{1}\right)=\bar{Y}^{2}\left(1-\frac{A_{4}^{2}}{A_{1}}\right)
$$

It follows from (2.19) and (2.20) that

$$
\begin{aligned}
A R B_{0}(T) & =\left|\frac{B_{0}\left(T_{1}\right)}{\bar{Y}}\right| \\
& =\frac{R M S E_{\text {min }}\left(T_{1}\right)}{\bar{Y}^{2}}=\left(1-\frac{A_{4}^{2}}{A_{1}}\right)
\end{aligned}
$$

Now, we state the following theorem.

THEOREM 2.2 -To the first degree of approximation,

$$
M S E_{\min }\left(T_{1}\right) \geq \bar{Y}^{2}\left(1-\frac{A_{4}^{2}}{A_{1}}\right)
$$

with equality holding if

$$
\alpha_{1}=\alpha_{10}^{*} \text {. }
$$

Inserting $\left(\alpha_{1}, \alpha_{2}\right)=(1,0)$ in $(2.1)$ and $\alpha_{1}=1$ in (2.15) we get a subclass of estimators from $T$ and $T_{1}$ as

$$
T_{1(1)}=\bar{y}\left\{\frac{(1-\beta) \bar{x}+\beta \bar{X}}{\beta \bar{x}+(1-\beta)}\right\}^{\delta}
$$

respectively to the first degree of approximation, as

$$
\begin{gathered}
B\left(T_{1(1)}\right)=\bar{Y} \frac{(1-f)}{2 n} \delta(1-2 \beta) C_{x}^{2}[\delta(1-2 \beta)+2 C-1], \\
M S E_{\min }\left(T_{1(1)}\right)=\bar{Y}^{2} \frac{(1-f)}{n}\left[C_{y}^{2}+\delta(1-2 \beta) C_{x}^{2}\{\delta(1-2 \beta)+2 C\}\right], \\
=\bar{Y}^{2}\left(1+A_{1}-2 A_{4}\right) .
\end{gathered}
$$

From (2.20) and (2.24) we have

$$
M S E_{\text {min }}\left(T_{1(1)}\right)-M S E_{\text {min }}\left(T_{1}\right)=\bar{Y}^{2} \frac{\left(A_{1}-A_{4}\right)^{2}}{A_{1}}>0
$$

which follows that the proposed $T_{1}$-family of estimators is more efficient than the $T_{1(1)}$-sub family of estimators. 
From (2.10) and (2.20) we have

$$
M S E_{\text {min }}\left(T_{1}\right)-M S E_{\text {min }}(T)=\frac{\bar{Y}^{2}\left(A_{1} A_{5}-A_{3} A_{4}\right)^{2}}{A_{1}\left(A_{1} A_{2}-A_{3}^{2}\right)} \geq 0 .
$$

It follows from (2.22) that the proposed $T$-family of estimators is more efficient than the $T_{1}$-subfamily of estimators. Hence the proposed family of estimators $T$ is more efficient than the Searls (1964) estimator $\bar{y}_{S e}$ and Srivastava (1964) and Prasad (1989) estimator $\bar{y}_{S P}$.

\section{A THREE PARAMETER RATIO-PRODUCT- RATIO ESTIMATOR}

If we set $\alpha_{1}=\alpha$ (a constant, say) and $\alpha_{2}=(1-\alpha)$ [i.e. when the sum of weights in (2.1) is unity $\left.\Rightarrow \alpha_{1}+\alpha_{2}=1\right]$ in (2.1) we get a three parameter ratio-product-ratio estimator for population mean $\bar{Y}$ as

$$
T_{(\alpha, \beta)}^{(\delta)}=\bar{y}\left[\alpha\left\{\frac{(1-\beta) \bar{x}+\beta \bar{X}}{\beta \bar{x}+(1-\beta) \bar{X}}\right\}^{\delta}+(1-\alpha)\left\{\frac{\beta \bar{x}+(1-\beta) \bar{X}}{(1-\beta) \bar{x}+\beta \bar{X}}\right\}\right] .
$$

Some members of the proposed sub-class of estimators $T_{(\alpha, \beta)}^{(\delta)}$ are shown in Table 3.1 .

Table 3.1-Some members of the sub-class of estimators $T_{(\alpha, \beta)}^{(\delta)}=T_{(k)}^{*}$ are listed in Table 3.1

\begin{tabular}{|c|c|c|c|c|}
\hline \multirow{2}{*}{ S.no } & Estimator & \multicolumn{3}{|c|}{ Values of } \\
& & & \multicolumn{3}{|c|}{ constant } \\
\cline { 3 - 7 } & $T_{(1)}^{*}=\bar{y}$ & $1 / 2$ & $1 / 2$ & 1 \\
\hline 2. & $T_{(2)}^{*}=\bar{y}\left(\frac{\bar{X}}{\bar{x}}\right)$ & 1 & 1 & 1 \\
\hline 3. & $T_{(3)}^{*}=\bar{y}\left(\frac{\bar{x}}{\bar{X}}\right)$ & 0 & 1 & 0 \\
\hline 4. & $T_{(4)}^{*}=\bar{y}\left(\frac{\bar{X}}{\bar{x}}\right)^{1 / 2}$ & 1 & 1 & $1 / 2$ \\
\hline
\end{tabular}




\begin{tabular}{|c|c|c|c|c|}
\hline \multirow[t]{2}{*}{ S.no } & \multirow[t]{2}{*}{ Estimator } & \multicolumn{3}{|c|}{$\begin{array}{l}\text { Values of } \\
\text { constant }\end{array}$} \\
\hline & & $\alpha$ & $\beta$ & $\delta$ \\
\hline 5. & $\begin{array}{l}\qquad T_{(5)}^{*}=\bar{y}\left(\frac{\bar{X}}{\bar{x}}\right)^{2} \\
\text { Kadilar and Cingi (2006) }\end{array}$ & 1 & 1 & 2 \\
\hline 6. & $T_{(6)}^{*}=\bar{y}\left\{\frac{\left(1-C_{x}\right) \bar{x}+C_{x} \bar{X}}{C_{x} \bar{x}+\left(1-C_{x}\right) \bar{X}}\right\}^{1 / 2}$ & 1 & $C_{\mathrm{x}}$ & $1 / 2$ \\
\hline 7. & $T_{(7)}^{*}=\bar{y}\left\{\frac{(1-\rho) \bar{x}+\rho \bar{X}}{\rho \bar{x}+(1-\rho) \bar{X}}\right\}^{1 / 2}$ & 1 & $\rho$ & $1 / 2$ \\
\hline 8. & $T_{(8)}^{*}=\bar{y}\left\{\frac{(1-\rho) \bar{x}+\rho \bar{X}}{\rho \bar{x}+(1-\rho) \bar{X}}\right\}^{1 / 2}$ & 1 & $\rho$ & 1 \\
\hline 9. & $T_{(9)}^{*}=\bar{y}\left\{\frac{\left(1-C_{y}\right) \bar{x}+C_{y} \bar{X}}{C_{y} \bar{x}+\left(1-C_{y}\right) \bar{X}}\right\}^{1 / 2}$ & 1 & $C_{\mathrm{y}}$ & $1 / 2$ \\
\hline 10. & $T_{(10)}^{*}=\bar{y}\left\{\frac{\left(1-C_{y}\right) \bar{x}+C_{y} \bar{X}}{C_{y} \bar{x}+\left(1-C_{y}\right) \bar{X}}\right\}$ & 1 & $C_{\mathrm{y}}$ & 1 \\
\hline 11. & $T_{(11)}^{*}=\bar{y}\left\{\frac{\left(1-C_{y}\right) \bar{x}+C_{y} \bar{X}}{C_{y} \bar{x}+\left(1-C_{y}\right) \bar{X}}\right\}^{2}$ & 1 & $C_{\mathrm{y}}$ & 2 \\
\hline 12. & $T_{(12)}^{*}=\bar{y}\left\{\frac{(1-C) \bar{x}+C \bar{X}}{C \bar{x}+(1-C) \bar{X}}\right\}^{1 / 2}$ & 1 & $C$ & $1 / 2$ \\
\hline 13. & $T_{(13)}^{*}=\bar{y}\left\{\frac{(1-C) \bar{x}+C \bar{X}}{C \bar{x}+(1-C) \bar{X}}\right\}^{1 / 2}$ & 1 & $C$ & 1 \\
\hline 14. & $T_{(13)}^{*}=\bar{y}\left\{\frac{(1-C) \bar{x}+C \bar{X}}{C \bar{x}+(1-C) \bar{X}}\right\}^{2}$ & 1 & $C$ & 2 \\
\hline 15. & $T_{(15)}^{*}=\frac{\bar{y}}{2}\left\{\left(\frac{\bar{X}}{\bar{x}}\right)^{2}+\left(\frac{\bar{x}}{\bar{X}}\right)\right\}$ & $1 / 2$ & 1 & 2 \\
\hline
\end{tabular}




\begin{tabular}{|c|c|c|c|c|c|}
\hline \multirow[t]{2}{*}{ S.no } & \multirow{2}{*}{\multicolumn{2}{|c|}{ Estimator }} & \multicolumn{3}{|c|}{$\begin{array}{l}\text { Values of } \\
\text { constant }\end{array}$} \\
\hline & & & $\alpha$ & $\beta$ & $\delta$ \\
\hline 16. & $T_{(16)}^{*}=\frac{\bar{y}}{2}\left[\left\{\frac{\left(1-C_{x}\right) \bar{x}+C_{x} \bar{X}}{C_{x} \bar{x}+\left(1-C_{x}\right) \bar{X}}\right\}^{2}+\left\{\frac{C_{x} \bar{x}+\left(1-C_{x}\right) \bar{X}}{\left(1-C_{x}\right) \bar{x}+C_{x} \bar{X}}\right.\right.$ & & $1 / 2$ & $C_{\mathrm{x}}$ & 2 \\
\hline 17. & $T_{(17)}^{*}=\frac{\bar{y}}{2}\left[\left\{\frac{(1-\rho) \bar{x}+\rho \bar{X}}{\rho \bar{x}+(1-\rho) \bar{X}}\right\}^{2}+\left\{\frac{\rho \bar{x}+(1-\rho) \bar{X}}{(1-\rho) \bar{x}+\rho \bar{X}}\right\}\right.$ & & $1 / 2$ & $\rho$ & 2 \\
\hline 18. & $T_{(18)}^{*}=\frac{\bar{y}}{2}\left[\left\{\frac{\left(1-C_{y}\right) \bar{x}+C_{y} \bar{X}}{C_{y} \bar{x}+\left(1-C_{y}\right) \bar{X}}\right\}^{2}+\left\{\frac{C_{y} \bar{x}+\left(1-C_{y}\right) \bar{X}}{\left(1-C_{y}\right) \bar{x}+C_{y} \bar{X}}\right.\right.$ & & $1 / 2$ & $C_{\mathrm{y}}$ & 2 \\
\hline 19. & $T_{(19)}^{*}=\frac{\bar{y}}{2}\left[\left\{\frac{(1-C) \bar{x}+C \bar{X}}{C \bar{x}+(1-C) \bar{X}}\right\}^{2}+\left\{\frac{C \bar{x}+(1-C) \bar{X}}{(1-C) \bar{x}+C \bar{X}}\right\}\right.$ & & $1 / 2$ & $C$ & 2 \\
\hline 20. & $\begin{array}{c}T_{(20)}^{*}=\bar{y}\left(\frac{\bar{X}}{\bar{x}}\right)^{\delta}=\bar{y}_{s} \\
\text { Srivastava (1967) estimator }\end{array}$ & & 1 & 1 & $\delta$ \\
\hline 21. & $\begin{aligned} T_{(21)}^{*}=\bar{y}\left[\alpha\left(\frac{\bar{X}}{\bar{x}}\right)^{\delta}+(1-\alpha)\left(\frac{\bar{X}}{\bar{x}}\right)\right]=\bar{y}_{G S} \\
\text { Pandey(1980) estimator }\end{aligned}$ & & $\alpha$ & 1 & $\delta$ \\
\hline 22. & $\begin{array}{l}T_{(22)}^{*}=\bar{y}\left[\alpha\left(\frac{\bar{X}}{\bar{x}}\right)+(1-\alpha)\left(\frac{\bar{x}}{\bar{X}}\right)\right]=\bar{y}_{S R} \\
\text { Singh and Ruiez Espejo(2003)estimator }\end{array}$ & & $\alpha$ & 1 & 1 \\
\hline 23. & $\begin{array}{c}T_{(23)}^{*}=\bar{y}\left[\alpha\left\{\frac{(1-\beta) \bar{x}+\beta \bar{X}}{\beta \bar{x}+(1-\beta) \bar{X}}\right\}+(1-\alpha)\left\{\frac{\beta \bar{x}+(1-\beta) \bar{X}}{(1-\beta) \bar{x}+\beta \bar{X}}\right\}\right] \\
\text { Chami et al (2012) estimator }\end{array}$ & $=\bar{y}_{C S T}$ & $\alpha$ & $\beta$ & 1 \\
\hline
\end{tabular}

Inserting $\alpha_{1}=\alpha$ and $\alpha_{2}=(1-\alpha)$ in (2.5) and (2.7) we get the bias and MSE of $T_{(\alpha, \beta)}^{(\delta)}$ to the first degree of approximation, respectively, as 


$$
\begin{gathered}
B\left(T_{(\alpha, \beta)}^{(\delta)}\right)=\bar{Y} \frac{(1-f)(1-2 \beta)}{n} C_{x}^{2}\left[\begin{array}{c}
\frac{\alpha \delta^{2}(1-2 \beta)}{2}+\alpha\left\{\beta-\frac{(\delta+2)}{2}\right\} \\
-C\{1-\alpha(\delta+1)\}-\beta+1
\end{array}\right] \\
\operatorname{MSE}\left(T_{(\alpha, \beta)}^{\delta}\right)=\frac{(1-f)}{n} \bar{Y}^{2}\left[C_{y}^{2}+C_{x}^{2}(1-2 \beta)(1-\alpha(1+\delta))\{(1-2 \beta)(1-\alpha(1+\delta))-2 C\}\right] .
\end{gathered}
$$

Taking for gradient $\nabla=\left(\frac{\partial}{\partial \alpha}, \frac{\partial}{\partial \beta}\right)$ of (3.3), we get

$$
\nabla M S E\left(T_{(\alpha, \beta)}^{(\delta)}\right)=2(1+\delta) \frac{(1-f)}{n} \bar{Y}^{2} C_{x}^{2}[\{1-\alpha(1+\delta)\}(1-2 \beta)-C](1-2 \beta,(1-\alpha(1+\delta))) .
$$

Putting (3.4) to zero to obtain the critical points, we obtain the following solutions:

$$
\alpha=\frac{1}{(1+\delta)}, \beta=\frac{1}{2}
$$

or

$$
C=\{1-\alpha(1+\delta)\}(1-2 \beta)
$$

We now calculate the minimal value of the MSE. Putting (3.5) into the estimator $T_{(\alpha, \beta)}^{(\delta)}$ yields the unbiased estimator $\bar{y}$ (sample mean) of the population mean $\bar{Y}$. Thus, we arrive at the mean squared error of the sample mean:

$$
\operatorname{MSE}\left(T_{\left(\frac{1}{1+\delta}, \frac{1}{2}\right)}^{(\delta)}\right)=\operatorname{MSE}(\bar{y})=\frac{(1-f)}{n} \bar{Y}^{2} C_{y}^{2}=\frac{(1-f)}{n} S_{y}^{2} .
$$

Inserting (3.6) into the estimator, an asymptotically optimum estimator (AOE) $T_{(\alpha, \beta)}^{(0)}$ is found. To the first degree of approximation, the MSE of the AOE $T_{(\alpha, \beta)}^{(0)}$ is given by

$$
\begin{aligned}
\operatorname{MSE}\left(T_{(\alpha, \beta)}^{(0)}\right) & =\frac{(1-f)}{n} \bar{Y}^{2}\left(C_{y}^{2}-C^{2} C_{x}^{2}\right), \\
& =\frac{(1-f)}{n} S_{y}^{2}\left(1-\rho^{2}\right) .
\end{aligned}
$$

That is, the same mean squared error as found in Srivastava (1967), Pandey (1980), Singh and Ruiz Espejo (2003) and Chami et al (2012). In fact, Srivastava (1971, 
1980) have proved that the $M S E$ of $T_{(\alpha, \beta)}^{(0)}$ in (3.8) is the minimal possible mean squared error up to first degree of approximation for a wide class of estimators to which the estimator (3.3) also belongs, for instance, for estimators of the form $T_{g}=\bar{y} \cdot g(\bar{x} / \bar{X})$, where $g$ is a $C^{2}$ - function with $g(1)=1$. Thus, whatever value $\mathrm{C}$ has, we are always able to choose an $\operatorname{AOE} T_{(\alpha, \beta)}^{(0)}$ from the three parameter family in (3.1).

\section{EFFICIENCY COMPARISONS}

Inserting $\alpha_{1}=\alpha$ (say, a constant) and $\alpha_{2}=(1-\alpha)$ in (2.5) we get the MSE of the proposed estimator $T_{(\alpha, \beta)}^{(\delta)}$ to the first degree of approximation, as

$$
\operatorname{MSE}\left(T_{(\alpha, \beta)}^{(\delta)}\right)=\bar{Y}^{2}\left[1+A_{2}-2 A_{5}+\alpha^{2}\left(A_{1}+A_{2}-2 A_{3}\right)-2 \alpha\left(A_{2}-A_{3}+A_{4}-A_{5}\right)\right]
$$

which is minimum when

$$
\alpha=\frac{\left(A_{2}-A_{3}+A_{4}-A_{5}\right)}{\left(A_{1}+A_{2}-2 A_{3}\right)}=\alpha_{\text {opt }} \text { (say). }
$$

Thus the resulting minimum MSE of $T_{(\alpha, \beta)}^{(\delta)}$ is given by

$$
\begin{aligned}
& \min \cdot \operatorname{MSE}\left(T_{(\alpha, \beta)}^{(\delta)}\right)=\bar{Y}^{2}\left[1+A_{2}-2 A_{5}-\frac{\left(A_{2}-A_{3}+A_{4}-A_{5}\right)^{2}}{\left(A_{1}+A_{2}-2 A_{3}\right)}\right] \\
& \min . \operatorname{MSE}\left(T_{(\alpha, \beta)}^{(\delta)}\right)=\frac{(1-f)}{n} \bar{Y}^{2} C_{y}^{2}\left(1-\rho^{2}\right)
\end{aligned}
$$

which equals to the approximate variance / $M S E$ of the usual linear regression estimator $\bar{y}_{l r}$ i.e.

$$
\min . M S E\left(T_{(\alpha, \beta)}^{(\delta)}\right)=\operatorname{MSE}\left(\bar{y}_{l r}\right)=\frac{(1-f)}{n} \bar{Y}^{2} C_{y}^{2}\left(1-\rho^{2}\right)=\frac{(1-f)}{n} S_{y}^{2}\left(1-\rho^{2}\right) .
$$

Now from (1.2), (1.5), (1.6) and (4.4) we have

$$
\operatorname{MSE}(\bar{y})-\min . . \operatorname{MSE}\left(T_{(\alpha, \beta)}^{(\delta)}\right)=\frac{(1-f)}{n} \bar{Y}^{2} C_{y}^{2} \rho^{2} \geq 0,
$$




$$
\begin{aligned}
& \operatorname{MSE}\left(\bar{y}_{R}\right)-\min . . \operatorname{MSE}\left(T_{(\alpha, \beta)}^{(\delta)}\right)=\frac{(1-f)}{n} \bar{Y}^{2} C_{x}^{2}(1-C)^{2} \geq 0, \\
& \operatorname{MSE}\left(\bar{y}_{p}\right)-\min \cdot \operatorname{MSE}\left(T_{(\alpha, \beta)}^{(\delta)}\right)=\frac{(1-f)}{n} \bar{Y}^{2} C_{x}^{2}(1+C)^{2} \geq 0 .
\end{aligned}
$$

It follows from (4.5) to (4.7) that the suggested estimator $T_{(\alpha, \beta)}^{(\delta)}$ is more efficient than :

(i) the usual unbiased estimator $\bar{y}$,

(ii) the usual ratio estimator $\bar{y}_{R}$ except when $C \neq 1$, the case where both the estimators $\bar{y}_{R}$ and $T_{(\alpha, \beta)}^{(\delta)}$ are equally efficient.

(iii) the usual product estimator $\bar{y}_{p}$ except when $C \neq-1$, the case where both the estimators $\bar{y}_{P}$ and $T_{(\alpha, \beta)}^{(\delta)}$ are equally efficient.

Expression (4.4) shows that the suggested estimator $T_{(\alpha, \beta)}^{(\delta)}$ is at par with the regression estimator $\bar{y}_{l r}$.

From (2.10) and (4.3) we have

$\min . \operatorname{MSE}\left(T_{(\alpha, \beta)}^{(\delta)}\right)-\min \cdot \operatorname{MSE}(T)=\left[\frac{\bar{Y}^{2}\left[A_{1}\left(A_{2}-A_{5}\right)+A_{4}\left(A_{3}-A_{2}\right)+A_{3}\left(A_{5}-A_{3}\right)\right]^{2}}{\left(A_{1}+A_{2}-2 A_{3}\right)\left(A_{1} A_{2}-A_{3}^{2}\right)}\right]>0$

It follows that the suggested $T$ - family of estimators is more efficient than the members of $T_{(\alpha, \beta)}^{(\delta)}$ - family of estimators.

Hence the proposed family of estimators $T$ is more efficient than the usual unbiased estimator $\bar{y}$, ratio estimator $\bar{y}_{R}$, product estimator $\bar{y}_{p}$, usual regression estimator $\bar{y}_{l r}$ and the two parameter ratio-product-ratio estimator $\bar{y}_{C S T}$ due to Chami et al (2012). 
4.1. Comparing the MSE of the sample mean estimator $\bar{y}$ to the suggested estimator $T_{(\alpha, \beta)}^{(\delta)}$

It is known that the sample mean estimator $\bar{y}$ is preferred for $-\frac{1}{2} \leq C \leq \frac{1}{2}$.

Then from (1.2) and (3.3) we have

$$
\operatorname{MSE}(\bar{y})-\operatorname{MSE}\left(T_{(\alpha, \beta)}^{(\delta)}\right)=\frac{(1-f)}{n} \bar{Y}^{2} C_{x}^{2}(1-2 \beta)\{1-\alpha(1+\delta)\}[2 C-\{1-\alpha(1+\delta)\}(1-2 \beta)]
$$

which is positive if

$$
(1-2 \beta)\{1-\alpha(1+\delta)\}[2 C-\{1-\alpha(1+\delta)\}(1-2 \beta)]>0
$$

Therefore, either

(i)

$$
\alpha>\frac{1}{(1+\delta)}, \beta>\frac{1}{2} \text { and } C>\frac{\{1-\alpha(1+\delta)\}(1-2 \beta)}{2}
$$

(ii) $\quad \alpha<\frac{1}{(1+\delta)}, \beta>\frac{1}{2}$ and $C<\frac{\{1-\alpha(1+\delta)\}(1-2 \beta)}{2}$

(iii) $\quad \alpha>\frac{1}{(1+\delta)}, \beta<\frac{1}{2}$ and $C<\frac{\{1-\alpha(1+\delta)\}(1-2 \beta)}{2}$, or

(iv) $\quad \alpha<\frac{1}{(1+\delta)}, \beta<\frac{1}{2}$ and $C>\frac{\{1-\alpha(1+\delta)\}(1-2 \beta)}{2}$.

Combining these with the condition $-\frac{1}{2} \leq C \leq \frac{1}{2}$, we get the following explicit ranges.

(i) If $0<C \leq \frac{1}{2}$ and $\beta>\frac{1}{2}$, then $\frac{1}{2}<\alpha<\frac{(2 \beta+2 C-1)}{(1+\delta)(2 \beta-1)}$ (from (i) )

(ii) If $0<C \leq \frac{1}{2}$ and $\beta<\frac{1}{2}$, then $\frac{(2 \beta+2 C-1)}{(1+\delta)(2 \beta-1)}<\alpha<\frac{1}{(1+\delta)}$ (from (iv) )

(iii) If $-\frac{1}{2} \leq C<0$ and $\beta>\frac{1}{2}$, then $\frac{(2 \beta+2 C-1)}{(1+\delta)(2 \beta-1)}<\alpha<\frac{1}{(1+\delta)}$ (from (ii) )

(iv) If $-\frac{1}{2} \leq C<0$ and $\beta<\frac{1}{2}$, then $\frac{1}{(1+\delta)}<\alpha<\frac{(2 \beta+2 C-1)}{(1+\delta)(2 \beta-1)}$ ( from (iii) ) 
Thus the proposed estimator $T_{(\alpha, \beta)}^{(\delta)}$ is more efficient than the sample mean $\bar{y}$ as long as either of the conditions (i) to (iv) is satisfied.

4.2. Comparing the MSE of the ratio estimator $\bar{y}_{R}$ to the suggested estimator $T_{(\alpha, \beta)}^{(\delta)}$.

For $C>\frac{1}{2}$, the ratio estimator $\bar{y}_{R}$ is used instead of the sample mean $\bar{y}$ or product estimator $\bar{y}_{p}$. We are interested in finding out a range of plausible values for $\alpha$ and $\beta$ in which the suggested estimator $T_{(\alpha, \beta)}^{(\delta)}$ better than the ratio estimator $\bar{y}_{R}$ Taking the difference of (1.5) and (3.3), we have

$$
\begin{array}{r}
\operatorname{MSE}\left(\bar{y}_{R}\right)-\operatorname{MSE}\left(T_{(\alpha, \beta)}^{(\delta)}\right)=\frac{(1-f)}{n} \bar{Y}^{2} C_{x}^{2}[2 \alpha \beta(1+\delta)-\alpha(1+\delta)-2 \beta] \\
{[2(C-1)-\{2 \alpha \beta(1+\delta)-\alpha(1+\delta)-2 \beta\}]}
\end{array}
$$

which is non-negative if

$$
[2 \alpha \beta(1+\delta)-\alpha(1+\delta)-2 \beta][2(C-1)-\{2 \alpha \beta(1+\delta)-\alpha(1+\delta)-2 \beta\}]>0
$$

i.e. if $\left[\alpha \beta(1+\delta)-\frac{\alpha(1+\delta)}{2}-\beta\right]\left[C-1-\left\{\alpha \beta(1+\delta)-\frac{\alpha(1+\delta)}{2}-\beta\right\}\right]>0$

Therefore,

(i) $C-1>\left[\alpha \beta(1+\delta)-\frac{\alpha(1+\delta)}{2}-\beta\right]>0$ or

(ii) $C-1<\left[\alpha \beta(1+\delta)-\frac{\alpha(1+\delta)}{2}-\beta\right]<0$

Hence, from solution (i), where $C>1$, we have the following

(i) If $\beta<\frac{1}{2}$, then $\frac{2(\beta+C-1)}{(1+\delta)(2 \beta-1)}<\alpha<\frac{2 \beta}{(1+\delta)(2 \beta-1)}$.

(ii) If $\beta>\frac{1}{2}$, then $\frac{(\beta+C-1)}{(1+\delta)(2 \beta-1)}<\alpha<\frac{2 \beta}{(1+\delta)(2 \beta-1)}$.

Further, from solution (ii), where $\frac{1}{2}<C<1$, we obtain the following. 
(i) If $\beta<\frac{1}{2}$, then $\frac{2 \beta}{(1+\delta)(2 \beta-1)}<\alpha<\frac{2(\beta+C-1)}{(1+\delta)(2 \beta-1)}$.

(ii) If $\beta>\frac{1}{2}$, then $\frac{(\beta+C-1)}{(1+\delta)(2 \beta-1)}<\alpha<\frac{2 \beta}{(1+\delta)(2 \beta-1)}$.

4.3. Comparing the MSE of the product estimator to the proposed estimator $T_{(\alpha, \beta)}^{(\delta)}$

It is known that, for $C<-\frac{1}{2}$, the product estimator $\bar{y}_{p}$ is preferred to the sample mean estimator $\bar{y}$ and ratio estimator $\bar{y}_{R}$. Therefore, we seek a range of $\alpha$ and $\beta$ values where the proposed estimator $T_{(\alpha, \beta)}^{(\delta)}$ has fewer MSE than the usual product estimator $\bar{y}_{p}$.

From (1.4) and (3.3) we have

$$
\begin{aligned}
\operatorname{MSE}\left(\bar{y}_{p}\right)-\operatorname{MSE}\left(T_{(\alpha, \beta)}^{(\delta)}\right)= & \frac{(1-f)}{n} \bar{Y}^{2} C_{x}^{2}[2+2 \alpha \beta(1+\delta)-\alpha(1+\delta)-2 \beta] \\
& {[2 C-\{2 \alpha \beta(1+\delta)-\alpha(1+\delta)-2 \beta\}] } \\
= & \frac{(1-f)}{n} \bar{Y}^{2} C_{x}^{2}\left[1+\alpha \beta(1+\delta)-\frac{\alpha(1+\delta)}{2}-\beta\right] \\
& {\left[C-\left\{\alpha \beta(1+\delta)-\frac{\alpha(1+\delta)}{2}-\beta\right\}\right] }
\end{aligned}
$$

which is greater than zero if

$$
\left[1+\alpha \beta(1+\delta)-\frac{\alpha(1+\delta)}{2}-\beta\right]\left[C-\left\{\alpha \beta(1+\delta)-\frac{\alpha(1+\delta)}{2}-\beta\right\}\right]>0
$$

We obtain following two cases:

(i) $C>\left[\alpha \beta(1+\delta)-\frac{\alpha(1+\delta)}{2}-\beta\right]>-1$ (if both factors in (4.14) are non-negative)

(ii) $C<\left[\alpha \beta(1+\delta)-\frac{\alpha(1+\delta)}{2}-\beta\right]<-1$ (if both factors in (4.14) are negative)

Observing that $C<-\frac{1}{2}$, we get from (i) 


$$
-\frac{1}{2}>C>\left[\alpha \beta(1+\delta)-\frac{\alpha(1+\delta)}{2}-\beta\right]
$$

We note that this implies $-1<C<-\frac{1}{2}$ and the range for $\alpha$ and $\beta$ where these inequalities hold are explicitly given by the following two cases:

(i) If $\beta<\frac{1}{2}$, then $\frac{2(\beta+C)}{(1+\delta)(2 \beta-1)}<\alpha<\frac{2(\beta-1)}{(1+\delta)(2 \beta-1)}$,

(ii) If $\beta>\frac{1}{2}$, then $\frac{2(\beta-1)}{(1+\delta)(2 \beta-1)}<\alpha<\frac{2(\beta+C)}{(1+\delta)(2 \beta-1)}$.

In case (ii), where $C<-1$ (and hence $C<-\frac{1}{2}$ ), the following range for $\alpha$ and $\beta$ can be obtained.

(i) If $\beta<\frac{1}{2}$, then $\frac{(\beta-1)}{(1+\delta)(2 \beta-1)}<\alpha<\frac{(\beta+C)}{(1+\delta)(2 \beta-1)}$.

(ii) If $\beta>\frac{1}{2}$, then $\frac{(\beta+C)}{(1+\delta)(2 \beta-1)}<\alpha<\frac{(\beta-1)}{(1+\delta)(2 \beta-1)}$.

4.4. Comparing the MSE of Srivastava's (1967) Estimator $\bar{y}_{S}=\bar{y}\left(\frac{\bar{X}}{\bar{x}}\right)^{\delta}$ with the proposed estimator $T_{(\alpha, \beta)}^{(\delta)}$

Let $\delta$ be a preassigned constant. Then for the purpose of comparison, we write the MSE of the Srivastava's (1967) estimator $\bar{y}_{S}$ to the first degree of approximation as

$$
\operatorname{MSE}\left(\bar{y}_{S}\right)=\frac{(1-f)}{n} \bar{Y}^{2}\left[C_{y}^{2}+\delta(\delta-2 C) C_{x}^{2}\right]
$$

From (3.3) and (4.16) we have

$$
\begin{aligned}
& \operatorname{MSE}\left(\bar{y}_{S}\right)-\operatorname{MSE}\left(T_{(\alpha, \beta)}^{(\delta)}\right)=4 \frac{(1-f)}{n} \bar{Y}^{2} C_{x}^{2}\left[\alpha \beta(1+\delta)-\frac{\alpha(1+\delta)}{2}-\beta-\frac{(\delta-1)}{2}\right] \\
& {\left[C-\left\{\alpha \beta(1+\delta)-\frac{\alpha(1+\delta)}{2}-\beta+\frac{(1+\delta)}{2}\right\}\right] }
\end{aligned}
$$


which is positive if

$$
\left[\alpha \beta(1+\delta)-\frac{\alpha(1+\delta)}{2}-\beta-\frac{(\delta-1)}{2}\right]\left[C-\left\{\alpha \beta(1+\delta)-\frac{\alpha(1+\delta)}{2}-\beta+\frac{(1+\delta)}{2}\right\}\right]>0
$$

Therefore,
(i) $C-\frac{(1+\delta)}{2}>\left[\alpha \beta(1+\delta)-\frac{\alpha(1+\delta)}{2}-\beta\right]>\frac{(\delta-1)}{2}$,
(ii) $C-\frac{(1+\delta)}{2}<\left[\alpha \beta(1+\delta)-\frac{\alpha(1+\delta)}{2}\right]<\frac{(\delta-1)}{2}$.

Hence, from solution (i), where $C>\frac{(1+\delta)}{2}$, we have the following :

(i) If $\beta>\frac{1}{2}$, then $\frac{(2 \beta+\delta-1)}{(1+\delta)(2 \beta-1)}<\alpha<\frac{(2 \beta+2 C-\delta-1)}{(1+\delta)(2 \beta-1)}$,

(ii) If $\beta<\frac{1}{2}$, then $\frac{(2 \beta+2 C-\delta-1)}{(1+\delta)(2 \beta-1)}<\alpha<\frac{(2 \beta+\delta-1)}{(1+\delta)(2 \beta-1)}$.

Also, from solution (ii), where $\frac{1}{2}<C<1$, we obtain the following:

(i) If $\beta>\frac{1}{2}$, then $\frac{(2 \beta+2 C-\delta-1)}{(1+\delta)(2 \beta-1)}<\alpha<\frac{(2 \beta+\delta-1)}{(1+\delta)(2 \beta-1)}$,

(ii)If $\beta<\frac{1}{2}$, then $\frac{(2 \beta+\delta-1)}{(1+\delta)(2 \beta-1)}<\alpha<\frac{(2 \beta+2 C-\delta-1)}{(1+\delta)(2 \beta-1)}$.

4.5. Comparing the MSE of Pandey's (1980) estimator $\bar{y}_{G S}$ with the proposed estimator $T_{(\alpha, \beta)}^{(\delta)}$

Let $\alpha$ and $\delta$ be preassigned constants. Then to the first degree approximation, the MSE of the Pandey's (1980) estimator $\bar{y}_{G S}$ is given by

$$
\operatorname{MSE}\left(\bar{y}_{G S}\right)=\frac{(1-f)}{n} \bar{Y}^{2}\left[C_{y}^{2}+(1-\alpha(1+\delta)) C_{x}^{2}\{(1-\alpha(1+\delta))+2 C\}\right]
$$

From (3.3) and (4.18) we have 


$$
\begin{array}{r}
\operatorname{MSE}\left(\bar{y}_{G S}\right)-\operatorname{MSE}\left(T_{(\alpha, \beta)}^{(\delta)}\right)=4 \frac{(1-f)}{n} \bar{Y}^{2} C_{x}^{2}\{1-\alpha(\delta+1)\}(1-\beta) \\
{[\beta\{1-\alpha(\delta+1)\}+C]}
\end{array}
$$

which is non- negative if

$$
(1-\beta)\{1-\alpha(\delta+1)\}[\beta\{1-\alpha(\delta+1)+C\}]>0
$$

Therefore,

(i) $\beta<1, \alpha<\frac{1}{(1+\delta)}$ and $[\beta+C-\alpha \beta((1+\delta)]>0$

(ii) $\beta<1, \alpha>\frac{1}{(1+\delta)}$ and $[\beta+C-\alpha \beta((1+\delta)]<0$

(iii) $\beta>1, \alpha<\frac{1}{(1+\delta)}$ and $[\beta+C-\alpha \beta((1+\delta)]<0$

(iv) $\beta>1, \alpha>\frac{1}{(1+\delta)}$ and $[\beta+C-\alpha \beta((1+\delta)]>0$

Combining (i) and (iii) we get that the suggested estimator $T_{(\alpha, \beta)}^{(\delta)}$ is more efficient than the Pandey's (1980) estimator $\bar{y}_{G S}$ if

$$
\left.\begin{array}{ll}
\text { either } & -\frac{C}{\{1-\alpha(1+\delta)\}}<\beta<1, \alpha<\frac{1}{(1+\delta)} \\
\text { or } & 1<\beta<-\frac{C}{\{1-\alpha(1-\delta)\}}, \alpha<\frac{1}{(1+\delta)}
\end{array}\right\}
$$

Further, combining (ii) and (iv) we obtained that the proposed estimator $T_{(\alpha, \beta)}^{(\delta)}$ is more efficient than the Pandey's (1980) estimator $\bar{y}_{G S}$ if

$$
\left.\begin{array}{ll}
\text { either } & -\frac{C}{\{1-\alpha(1+\delta)\}}<\beta<1, \alpha>\frac{1}{(1+\delta)} \\
\text { or } & 1<\beta<-\frac{C}{\{1-\alpha(1+\delta)\}}, \alpha>\frac{1}{(1+\delta)}
\end{array}\right\}
$$


4.6. Comparing the MSE of the Chami et al (2012) estimator $T_{(23)}^{*}=\bar{y}_{C S T}$ to the suggested estimator $T_{(\alpha, \beta)}^{(\delta)}$

For the purpose of comparison of the suggested estimator $T_{(\alpha, \beta)}^{(\delta)}$ with the Chami et al (2012) estimator $T_{(23)}^{*}=\bar{y}_{C S T}$, we write the $M S E$ of $T_{(23)}^{*}$ to the first degree of approximation as

$$
\operatorname{MSE}\left(T_{(23)}^{*}\right)=\frac{(1-f)}{n} \bar{Y}^{2}\left[C_{y}^{2}+C_{x}^{2}(1-2 \alpha)(1-2 \beta)\{(1-2 \alpha)(1-2 \beta)-2 C\}\right]
$$

Let $(\alpha, \beta)$ be preassigned constants. Then from (3.3) and (4.22) we have

$$
\operatorname{MSE}\left(T_{(23)}^{*}\right)-\operatorname{MSE}\left(T_{(\alpha, \beta)}^{(\delta)}\right)=\frac{(1-f)}{n} \bar{Y}^{2} C_{x}^{2} \alpha(1-2 \beta)\left[\begin{array}{l}
2 \delta\{(1-2 \beta)(1-\alpha)+C\} \\
-\alpha(1-2 \beta) \delta^{2}-(2+2 C-3 \alpha)
\end{array}\right]
$$

which is non negative if

$$
\alpha(1-2 \beta)\left[2 \delta\{(1-2 \beta)(1-\alpha)+C\}-\alpha(1-2 \beta) \delta^{2}-(2+2 C-3 \alpha)\right]>0
$$

that is if

either $\left|\delta^{2} \alpha(1-2 \beta)-2 \delta\{(1-2 \beta)(1-\alpha)+C\}+(2+2 C-3 \alpha)\right|<0 ; \alpha(1-2 \beta)>0$

or $\left[\delta^{2} \alpha(1-2 \beta)-2 \delta\{(1-2 \beta)(1-\alpha)+C\}+(2+2 C-3 \alpha)\right]>0 ; \alpha(1-2 \beta)<0$

From (4.24) we note that the proposed estimator $T_{(\alpha, \beta)}^{(\delta)}$ is more efficient than the Chami et al (2012) estimator $T_{(23)}^{*}=\bar{y}_{C S T}$ if

(i) $\alpha>0, \beta<\frac{1}{2}$ and

$$
\left.\mid \delta^{2}(1-2 \beta)-2 \delta\{(1-2 \beta)(1-\alpha)+C\}+(2+2 C-3 \alpha)\right]<0
$$

(ii) $\alpha<0, \beta>\frac{1}{2}$ and

$$
\left.\mid \delta^{2}(1-2 \beta)-2 \delta\{(1-2 \beta)(1-\alpha)+C\}+(2+2 C-3 \alpha)\right]<0
$$

Further from (4.25) it is observed that the suggested estimator $T_{(\alpha, \beta)}^{(\delta)}$ is more efficient than the Chami et al (2012) estimator $T_{(23)}^{*}=\bar{y}_{C S T}$ if 
(i) $\alpha>0, \beta>\frac{1}{2}$ and

$$
\left\lfloor\delta^{2} \alpha(1-2 \beta)-2 \delta\{(1-2 \beta)(1-\alpha)+C\}+(2+2 C-3 \alpha)\right\rfloor>0
$$

(ii) $\alpha<0, \beta<\frac{1}{2}$ and

$$
\left\lfloor\delta^{2} \alpha(1-2 \beta)-2 \delta\{(1-2 \beta)(1-\alpha)+C\}+(2+2 C-3 \alpha)\right\rfloor>0
$$

\section{EMPIRICAL STUDY}

To see the merits of the members of the proposed class of estimators $T$ of the population mean $\bar{Y}$ over other existing estimators, we have considered a natural population data as earlier considered by Chami et al (2012).

The variables are:

$\bar{y}$ (study variate) : The maximum daily values (in feet) of groundwater at the site for the period from October 2009 to September 2010.

$\bar{x}$ (auxiliary variate) : The maximum daily values (in feet) of ground water for the period from October 2008 to September 2009.

The values of the required parameters are :

$\bar{Y}=0.5832, \bar{X}=0.6277, S_{y}=0.4480, S_{x}=0.7222, \rho=0.9125, C_{y}=0.7681$,

$C_{x}=1.1504$ and $C=0.6092, N=365, n=112$.

We have computed the percent relative efficiencies (PRES) of the suggested classes of estimators $T_{(\alpha, \beta)}^{(\delta)}, T_{1}$ and $T$ with respect to the usual unbiased estimator $\bar{y}$ by using the following formulae :

$\left.\operatorname{PRE}\left\{T_{(\alpha, \beta)}^{(\delta)}=T_{(k)}^{*}, \bar{y}\right\}=\frac{C_{y}^{2}}{\left[C_{y}^{2}+(1-2 \beta)^{2}(1-\alpha(1+\delta))^{2} C_{x}^{2}-2(1-2 \beta)(1-\alpha(1+\delta)) C C_{x}^{2}\right.}\right]^{* 100}$ $k=1$ to 19 


$$
\begin{aligned}
& \operatorname{PRE}\left(T_{1}=T_{1(l)}, \bar{y}\right)=\frac{\{(1-f) / n\} C_{y}^{2}}{\left[1-\frac{A_{4}^{2}}{A_{1}}\right]} * 100 \\
& l=1 \text { to } 10 \\
& \operatorname{PRE}\left(T=T_{(j)}, \bar{y}\right)=\frac{\{(1-f) / n\} C_{y}^{2}}{\left[1-\frac{\left(A_{2} A_{4}^{2}-2 A_{3} A_{4} A_{5}+A_{1} A_{5}^{2}\right)}{\left(A_{1} A_{2}-A_{3}^{2}\right)}\right]} * 100 \\
& j=1 \text { to } 12
\end{aligned}
$$

\begin{tabular}{|c|c|}
\hline \multicolumn{2}{|c|}{$\begin{array}{l}\text { PRES of members of the subclass of estimators } T_{(\alpha, \beta)}^{(\delta)}(\text { listed in Table 3.1) } \\
\text { with respect to the sample mean } \bar{y}\end{array}$} \\
\hline Estimator & $\operatorname{PRE}(., \bar{y})$ \\
\hline$T_{(1)}^{*}$ & 100.0000 \\
\hline$T_{(2)}^{*}$ & 196.0432 \\
\hline$T_{(3)}^{*}$ & 16.7329 \\
\hline$T_{(4)}^{*}$ & 514.7918 \\
\hline$T_{(5)}^{*}$ & 22.19005 \\
\hline$T_{(6)}^{*}$ & 583.7305 \\
\hline$T_{(7)}^{*}$ & 393.2448 \\
\hline$T_{(8)}^{*}$ & 367.6904 \\
\hline$T_{(9)}^{*}$ & 233.3748 \\
\hline$T_{(10)}^{*}$ & 557.2330 \\
\hline$T_{(11)}^{*}$ & 154.134 \\
\hline$T_{(12)}^{*}$ & 137.3067 \\
\hline
\end{tabular}

Findings are shown in Table 5.1 .

Table - 5.1- PREs of various estimators of population mean $\bar{Y}$ with respect to the sample mean $\bar{y}$ 


\begin{tabular}{l|l}
\hline$T_{(13)}^{*}$ & 196.0432 \\
\hline$T_{(14)}^{*}$ & 427.0306 \\
\hline$T_{(15)}^{*}$ & 514.7918 \\
\hline$T_{(16)}^{*}$ & 583.7305 \\
\hline$T_{(17)}^{*}$ & 393.2448 \\
\hline$T_{(18)}^{*}$ & 233.3748 \\
\hline$T_{(19)}^{*}$ & 137.3067 \\
\hline$T_{(20)}^{*}, T_{(21)}^{*}, T_{(22)}^{*}, T_{(23)}^{*}$ and $\bar{y}_{l r}$ & 597.5724 \\
\hline
\end{tabular}

PREs of members of the subclass of estimators $T_{1}$ (listed in Table 2.2)

with respect to the sample mean $\bar{y}$

\begin{tabular}{l|l}
\hline$T_{1(1)}$ & 515.9925 \\
\hline$T_{1(2)}$ & 198.7567 \\
\hline$T_{1(3)}$ & 586.6808 \\
\hline$T_{1(4)}$ & 393.9134 \\
\hline$T_{1(5)}$ & 371.1324 \\
\hline$T_{1(6)}$ & 558.7912 \\
\hline$T_{1(7)}$ & 156.5851 \\
\hline$T_{1(8)}$ & 137.6585 \\
\hline$T_{1(9)}$ & 196.4083 \\
\hline$T_{1(10)}$ & 427.8058 \\
\hline
\end{tabular}

PREs of members of the subclass of estimators $T$ (listed in Table 2.1) with respect to the sample mean $\bar{y}$

\begin{tabular}{l|l}
\hline$T_{(1)}$ & 602.8607 \\
\hline$T_{(2)}$ & 603.4527 \\
\hline$T_{(3)}$ & 602.8607 \\
\hline$T_{(4)}$ & 614.2654 \\
\hline$T_{(5)}$ & 732.8552 \\
\hline$T_{(6)}$ & 600.5716 \\
\hline$T_{(7)}$ & 648.1944 \\
\hline$T_{(8)}$ & 1120.9770 \\
\hline$T_{(9)}$ & 607.1476 \\
\hline$T_{(10)}$ & 605.0174 \\
\hline$T_{(11)}$ & 660.0981 \\
\hline
\end{tabular}




\begin{tabular}{l|l}
\hline$T_{(12)}$ & 611.0245 \\
\hline
\end{tabular}

It is observed from Table 5.1 that there is enough scope of selecting the values of the scalars:

(i) $(\alpha, \beta, \delta)$ to obtain estimators better than the usual unbiased estimator $\bar{y}$, ratio estimator $T_{(2)}^{*}=\bar{y}_{R}$, product estimator $T_{(3)}^{*}=\bar{y}_{p}$, Kadilar and Cingi's (2006) estimator $T_{(5)}^{*}$ from the three parameter estimator $T_{(\alpha, \beta)}^{(\delta)}$. We note that the estimator $T_{(15)}^{*}$ is at par with the Swain's ratio estimator $T_{(4)}^{*}$. However, the performance of the proposed estimator $T_{(16)}^{*}$ based on coefficient of variation $C_{\mathrm{x}}$ seems to be appreciable over the existing estimators $\bar{y}, T_{2}^{*}=\bar{y}_{R}, T_{3}^{*}=\bar{y}_{p}$ $, T_{(4)}^{*}, T_{(15)}^{*}$ and other members of the proposed class of estimators $T_{(\alpha, \beta)}^{(\delta)}$. The performance of the estimators $T_{(20)}^{*}, T_{(21)}^{*}, T_{(22)}^{*}, T_{(23)}^{*}$ due to Srivastava (1967), Pandey (1980), Singh and Ruiz Espejo (2003) and Chami et al (2012) at their optimum conditions are at par with the usual regression estimator $\bar{y}_{l r}$.

(ii) $(\delta, \beta)$ to obtain estimators better than the usual unbiased estimator $\bar{y}$, the ratio estimator $T_{(2)}^{*}=\bar{y}_{R}$, product estimator $T_{(3)}^{*}=\bar{y}_{p}$, from the proposed $T_{1}$ - family of estimators. Thus one can use the $T_{1}$ - family of estimators in practice for obtaining estimators better than the members of the three parameter $T_{(\alpha, \beta)^{-}}^{(\delta)}$ family of estimators.

(iii) $(\delta, \beta)$ to get estimators better than the usual unbiased estimator $\bar{y}$, the ratio estimator $T_{(2)}^{*}=\bar{y}_{R}$, product estimator $T_{(3)}^{*}=\bar{y}_{p}$, and the regression estimator $\bar{y}_{l r}$. We have generated twelve estimator $T_{(j)} ; j=1$ to 12 whose efficiency is larger than the regression estimator $\bar{y}_{l r}$. 
We also note that the members $T_{(j)} ; j=4,5,6,7,8,9,10,11,12$ are more efficient than the Upadhyaya and Singh (1985) estimators $T_{(1)}$ and $T_{(2)}$. Table 5.1 exhibits that the estimator $T_{(8)}$ based on coefficient of variation $C_{\mathrm{x}}$ has the largest efficiency $\left(\right.$ i.e. $\left.\operatorname{PRE}\left(T_{(8)}, \bar{y}\right)=1120.9770\right) \quad$ followed by the estimator $T_{5}$ (i.e. $\left.\operatorname{PRE}\left(T_{(5)}, \bar{y}\right)=732.8552\right)$.

Thus, in general, we conclude that the proposed family of estimators $T$ is to be preferred as it provides more efficient estimators as compared to the $T_{1}$ and $T_{(\alpha, \beta)}^{(\delta)}$ families of estimators.

\section{CONCLUSION}

In this article, we have considered the problem of estimating the population mean $\bar{Y}$ of the study variable $y$ using information on an auxiliary variable $x$. A class of estimators is suggested which includes the two-parameter ratio-product-ratio estimator recently proposed by Chami et al (2012). We have obtained the bias and mean squared error (MSE) of the suggested class of estimators up to first order of approximation. The merit of the suggested class of estimators is that it unifies several results at one place and also more efficient than the usual unbiased estimator, ratio estimator, regression estimator and Chami et al's (2012) estimator. Later we have suggested a three-parameter ratio-product-ratio estimator which also includes Chami et al's (2012) estimator and many other existing estimators. Which is at par with regression estimator. An empirical study is given in support of the present study. Both empirical and theoretical results show that the suggested class of estimators is better than the various existing estimators. Thus our recommendation is in the favour of the proposed class of estimators regarding its use in practice. 


\section{REFERENCES}

Chami, P.S., Sing, B. and Thomas, D. (2012): A Two-Parameter Ratio-Product-Ratio Estimator using Auxilary Information. ISRN Probability and Statistics, 2012, 1-15. DOI: $10.5402 / 2012 / 103860$.

Gupta, P.C. (1971): On Some ratio and product type estimations. Journal of Indian Society Agricultural Statistics, 23,111-112.

Kadilar, C. and Cingi, H. (2006): An improvement in estimating the population mean by using the correlation coefficient, Hacettepe Journal of Mathematics and Statistics, 35 (1), 103-109.

Murthy, M.N. (1964): Product Method of Estimation. Sankhya A, 26, 69-74.

Pandey, G.S. (1980): Product-cum-power estimators, Calcutta Statistical Association Bull., 29, 103-108.

Parsad, B. (1989): Some improved ratio type estimators of population mean and ratio in finite population sample surveys. Communication in Statistics - Theory and Method. 18(1): 379-392.

Reddy (1978): A study on the use of prior knowledge on certain population parameters in estimation. Sankhya,C, 40, 29-37.

Reddy, V.N. (1973): On ratio and product methods of estimation. Sankhaya,B 35(3): 307-316.

Reddy, V.N. (1974): On a transformed ratio method of estimation. Sankhaya, C 36(1): 59-70.

Sahai, A. and Ray, S.K. (1980): An efficient estimator using auxiliary information. Metrika 27(1): 271 275 .

Searls (1964): The utilization of known coefficient of variation in the estimation procedure. Journal of American Statistical Association, 59, 1125-1126.

Singh H.P. (1986): A generalized class of estimators of ratio, product and mean using supplementary information on auxiliary character in PPSWR sampling scheme. Gujarat Statistical Review, 13(2), 1-30.

Singh S. (2003): Advanced Sampling Theory with Applications. Kluwer Academic Publishers, The Netherlands.

Singh, H.P. and Ruiz-Espejo, M. (2003): On linear regression and ratio-product estimation of a finite population mean. The Statistician 52(1): 59-67.

Srivastava, S.K. (1967): An estimator using auxiliary information in sample surveys. Calcutta Statistical Association Bull. 16: 62-63.

Srivastava, S.K. (1971): A generalized estimator for the mean of a finite population using multi-auxiliary information. Journal of American Statistical Association, 66(334): 414-407.

Srivastava, S.K. (1974): On the ratio method of estimator. Tab Estadist Invest Opera 25,113-117.

Srivastava, S.K. (1980): A class of estimators using auxiliary information in sample surveys. The Canadian Journal of Statististics, 8(2): 253-254.

Swain, A.K.P.C. (2014): On an improved ratio type estimator of finite population mean in sample surveys. Revista Investigacion Operacional, 35(1), 49-57.

Upadhyaya, L.N., Singh, H.P., Vos, J.W.E. (1985): On the estimation of population means and ratios using supplementary information. Statistica Neerlandica 39(3), 309-318. 
Housila P. Singh

School of studies in Statistics

Vikram University, Ujjain- 456010, M.P., India

Email: hpsujn@gmail.com

\section{Anita Yadav}

School of studies in Statistics

Vikram University, Ujjain- 456010, M.P., India 